The effects of cancer and its treatment can have major adverse effects on the women it has affected and their families. This service has been set up with the support of Sutton Breast Friends and St Giles Hospice and will enable patients to discuss issues which were previously unrecognised relating to the impact on their altered body image, well-being and sexuality in a comfortable and open environment with a specialist nurse who has the skills to empower these patients to live their lives well, when suffering from the adverse effects of cancer and its treatment. Focus groups were utilised at the start to ensure that the service was set up how women wanted to ensure its success.

By developing this service and delivering this model of care, our aim is to:

- improve the wellbeing of women suffering with cancer and the adverse effects of its treatment

- enable women to take control and manage a range of problems impacting on their body image, sexuality and wellbeing

- enable wives/husbands/partners to discuss the issues and concerns relating to how cancer and its treatment may impact on their relationship

- prevent relationship disconnect linked to cancer and its treatments

There will continue to be an unmet need and these issues will remain unresolved for patients affecting their quality of life, and their ability to reconnect with their relationships. Evidence shows increased risk of relationship breakdown when suffering from the adverse effects of cancer and its treatment.

Due to this being a new service outcome measures will be collected and will be used to demonstrate the effect the intervention has had on patients self-worth, confidence and state of mind.

\section{INVESTING IN QUALITY IMPROVEMENT IN A HOSPICE - HOW IT HALVED WAITING TIMES AND IS CREATING A NEW CULTURE!}

Charles Daniels. St. Luke's Hospice Harrow

10.1136/bmjspcare-2018-ASPabstracts. 176

Over the last 2 years St Luke's Hospice has invested in the use a Quality Improvement Framework - The Model for Improvement (IHI), across the whole organisation.

This involved an investment in a QI lead, back filling the clinical time, actively supporting staff to participate and investment in a partnership with NIHR Collaboration for Leadership in Applied Health Research and Care (CLAHRC).

There have been 3 key interventions.

- Delivery of a QI programme to 3 cohorts of staff and volunteers.

- 33 people have attended the programme ( 2 doctors, 7 nurses, 4 Patient support, 1 therapist, 7 clinical support, 8 non-clinical and 4 volunteers), and 20 QI projects have been undertaken.

- The course has evolved and now has a more theory into practice, peer support, and trialling incremental improvement

- Investment in a partnership with the CLAHRC to improve service in our Woodgrange Centre
- We will show how we have improved our data collection

- Halving waiting times in Our Woodgrange Centre

- Invested in setting up the use of Care opinion as a way of improving the way we use patient stories to appreciate our staff and improve services.

This has been a process of parallel continuous learning. We will also share what we have learnt about leading such a process and what our staff have learnt along the way.

This will be helpful for anyone in a leadership role wanting to follow a similar approach.

\section{HOW WE HALVED THE WAITING TIME FOR HOSPICE SERVICE THROUGH IMPLEMENTING QI METHODOLOGY}

Charles Daniels, Caroline Cullen, Liz Evans, Rachel Mathews, Dannie Darko, Yewande Adeleke, Tom Woodcock. St. Luke's Hospice Harrow, CLAHRC NW London

\subsection{6/bmjspcare-2018-ASPabstracts.177}

As part of a wider QI programme St Luke's Hospice invested in a Partnership with NIHR Collaboration for Leadership in Applied Health Research and Care (CLAHRC) to assess the impact of applying Quality Improvement methodology to the Woodgrange Centre (WGC). The WGC is our Daycare and outpatient Centre.

The presentation will present the series of Quality improvement methodologies used and what we learnt from each step: These include:

- Initial engagement with patients, carers, staff, volunteers and referrers

- Touchpoint Mapping

- Using an action effect diagram to develop a shared aim and prioritise first steps

- Use of process mapping to engage staff in the change idea

- Introducing and supporting staff to try tests of change through PDSA cycles

- Learning to use measurement for improvement to demonstrate the improvements

We will show how this has changed the culture, enthused and developed our staff and amongst other improvements halved the average waiting time from referral to 1 st visit from 20 to 11 days.

We will share the added quantitative data to patient feedback. We now know that as well as patients reporting that the 'service is wonderful', 43\% believe the WGC has prevented a hospital admission.

\section{MONITORING CORTICOSTEROIDS: AN IMPROVEMENT PROJECT IN A SCOTTISH SPECIALIST PALLIATIVE CARE UNIT}

Claire McCullough. NHS Grampian

10.1136/bmjspcare-2018-ASPabstracts. 178

Background Corticosteroids are frequently prescribed in patients with advanced cancer for a wide range of indications. Many undesirable adverse effects are frequently reported including steroid-induced hyperglycaemia. For patients commenced on corticosteroids, without prior diagnosis of diabetes, twice weekly monitoring of capillary blood glucose(BM) is 